\title{
AVALIAÇÃO DA CONTINUIDADE ESPACIAL DE CARACTERÍSTICAS DENDROMÉTRICAS EM DIFERENTES IDADES DE POVOAMENTOS CLONAIS DE Eucalyptus sp. ${ }^{1}$
}

Honório Kanegae Junior² ${ }^{2}$ José Marcio de Mello³, José Roberto S. Scolforo ${ }^{3}$ e Antônio Donizette de Oliveira ${ }^{3}$

\begin{abstract}
RESUMO - O conhecimento da estrutura de continuidade espacial das características dendrométricas pode propiciar um eficiente controle de variação sobre as variáveis que se deseja estimar nos inventários sucessivos. Esse controle pode ser efetivado pelo uso de estratificadores baseados em mapas resultantes da krigagem estatística. Entretanto, o uso da krigagem carece de informações sobre a questão da continuidade espacial das variáveis descritoras dos povoamentos de Eucalyptus sp., assim como o comportamento dessas características no tempo. Este estudo teve como objetivo avaliar a estrutura de continuidade espacial de três características dendrométricas de 23 povoamentos clonais de Eucalyptus sp. em três medições sucessivas, localizados em regiões distintas do Estado de São Paulo. Para cada povoamento, variável e medição, ajustaram-se semivariogramas experimentais pelo método dos Quadrados Mínimos Ponderados. Através do grau de dependência espacial obtido pelos semivariogramas experimentais, avaliou-se o comportamento da continuidade espacial das variáveis. Mais de $70 \%$ dos povoamentoss analisados apresentaram de média a forte dependência espacial para volume e $80 \%$ para as variáveis área basal e altura dominante. $\mathrm{O}$ alcance das variáveis apresentou variações de 300 a $3.000 \mathrm{~m}$, dependendo do povoamento e da idade de medição. O porcentual de povoamentos com forte dependência espacial decresce com a idade, tendendo ao patamar de média dependência espacial, em todas as variáveis analisadas. O grau de dependência espacial ao longo de sucessivas medições variou de povoamento para povoamento e de medições para medições, podendo diminuir ou aumentar com o tempo, indicando que em cada povoamento e medição seja feita uma análise específica da continuidade espacial da variável de interesse.
\end{abstract}

Palavas-chave: Inventário florestal, geoestatística e continuidade espacial.

\section{EVALUATION OF SPATIAL CONTINUITY OF DENDROMETRIC CHARACTERISTICS OF CLONAL STANDS OF Eucalyptus sp. AT DIFFERENT AGES}

\begin{abstract}
The knowledge on the structure of spatial continuity of dendrometric characteristics can provide an efficient control of variation in the variables to be estimated in successive inventories. This control can be achieved by using of stratifiers based on maps from statistical kriging. However, kriging lacks information on the spatial continuity of descriptor variables for Eucalyptus sp. stands, as well as the behavior of this characteristic over time. This study evaluated the structure of spatial continuity for three dendrometric characteristics of 23 clonal stands of Eucalyptus sp. in 3 successive measurements, located in different areas of SP state. For each stand, variable and measurement, experimental semivariograms were adjusted by the weighted least squares method. Through the degree of spatial dependence obtained by the experimental semivariograms,
\end{abstract}

\footnotetext{
${ }^{1}$ Recebido em 30.08.2006 e aceito para publicação em 29.03.2007.

${ }^{2}$ VCP Florestal S/A. Rod. SP-255 Km 41,240, 14.210-000, Luiz Antônio-SP. E-mail: <honorio.kanegae@ vcp.com.br>.

${ }^{3}$ Departamento de Ciências Florestais da UFLA. Cx. Postal 37, 37.200-000 Lavras-MG. E-mail: <jmmello@ufla.br>; <scolforo@ufla.br>; <donizete@ufla.br>.
} 
the behavior of the spatial continuity of variables was evaluated. More than $70 \%$ of the analyzed stands showed average to strong spatial dependence for volume and $80 \%$ for the variables basal area and dominant height. The range of the variables showed variations from 300 to $3.000 \mathrm{~m}$, depending on the stand and age of measurement. The percentage of stands with strong spatial dependence decreases with age, tending to a sill of average spatial dependence, for all the analyzed variables. The degree of spatial dependence along successive measurements varies from stand to stand and from measurement to measurement, being likely to decrease or increase with time, suggesting a specific analysis of spatial dependence for the variable of interest for each stand and measurement.

Keywords: Forestry inventory, geoestatistic and spatial continuity.

\section{INTRODUÇÃO}

A estratificação nos inventários florestais tem forte influência sobre a precisão, tamanho da amostra (SCOLFORO e MELLO, 1997) e custos devido ao controle da variação que ela deve exercer sobre a variável de interesse a ser estimada. Normalmente, a estratificação da floresta é realizada com base em informações cadastrais, como a idade, a espécie, o espaçamento e o regime de manejo, embora o ideal fosse a estratificação baseada na própria variável de interesse.

A estatística clássica tem como pressuposto que as unidades amostrais são independentes e não apresentam dependência espacial. No entanto, a estatística espacial considera que os valores resultantes das parcelas estão associados à sua localização no espaço e, portanto, através de interpoladores é possível realizar a estimativa de pontos não amostrados. O melhor entendimento da interpolação espacial passa pelo conceito de vizinhança (ESRI, 2001). De acordo com esse conceito, coisas próximas são mais parecidas que outras mais distantes, portanto os valores medidos distantes dos locais a serem preditos têm pequeno relacionamento com os valores a serem estimados, tendendo a zero de influência.

Os interpoladores espaciais podem ser determinísticos e estatísticos. Os determinísticos são aqueles que geram estimativas a partir de pontos medidos e se baseiam na configuração espacial da amostra. Esses interpoladores não reconhecem agrupamentos de pontos, sendo os pesos proporcionais ao inverso da distância (YAMAMOTO, 2001). Para esses interpoladores, a redundância de informação dos pontos amostrados não é levada em consideração. Além do mais, esses estimadores não fornecem os erros associados a cada estimativa.
O interpolador geoestatístico, ou krigagem, utiliza propriedades estatísticas e estimativas da configuração espacial dos pontos amostrados para a estimativa do ponto desejado. A técnica de krigagem baseia-se no estudo da variabilidade espacial da característica de interesse. Essa técnica é superior aos demais interpoladores, por permitir calcular o erro associado a cada estimativa - variância da krigagem (JOURNEL e HUIJBREGTS, 1978). Assim, a geoestatística é uma técnica que merece estudos como uma possível e eficiente alternativa para controlar a variação na floresta. Como um dos produtos, pode-se considerar a elaboração de mapas, oriundos de interpolações espaciais, com estratos definidos nas primeiras medições do povoamento.

A abordagem de interpoladores espaciais, em especial a krigagem estatística, carece, entretanto, de informações sobre a questão da continuidade espacial das variáveis descritoras dos povoamentos de Eucalyptus sp., assim como o comportamento dessa característica no tempo. Dessa forma, os objetivos deste estudo foram analisar vários modelos de ajuste aplicáveis a diferentes fenômenos com continuidade espacial e avaliar o grau de dependência espacial de quatro características dendrométricas de 23 povoamentos clonais de Eucalyptus spp., em três diferentes idades e regiões do Estado de São Paulo.

\section{MATERIAL E MÉTODOS}

\subsection{Descrição da área de estudo}

Os dados deste estudo foram oriundos de florestas plantadas de Eucalyptus sp, em três regiões do Estado de São Paulo: Luiz Antônio, Vale do Paraíba e Capão Bonito (Figura 1). 


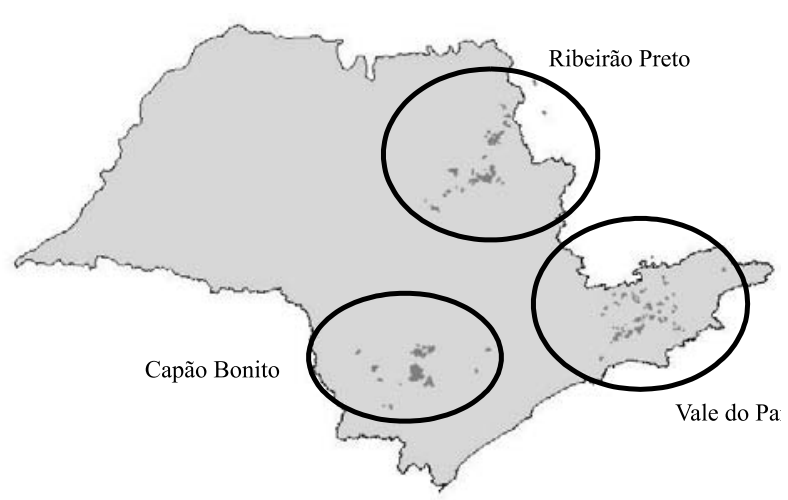

Figura 1 - Localização geográfica das regiões de estudo. Figure 1-Geographical location of the study areas.

Na região de Luiz Antônio, de acordo com Golfari et al. (1978) a precipitação média anual da região é de $1.000 \mathrm{~mm}$, temperatura média anual de $22^{\circ} \mathrm{C}$ e altitude de 970 m acima do nível do mar, e os solos predominantes são os Neossolos Quartzarênicos (areias quartzosas).

Na região do Vale do Paraíba, a precipitação média anual da região é de $1.200 \mathrm{~mm}$, temperatura média anual de $20{ }^{\circ} \mathrm{C}$ e altitude de $950 \mathrm{~m}$ acima do nível do mar, com predominância dos Argissolos (Podzólico Vermelho Amarelo).

A região de Capão Bonito é caracterizada por precipitação média anual da região de $1.600 \mathrm{~mm}$, temperatura média anual de $19{ }^{\circ} \mathrm{C}$ e altitude de $655 \mathrm{~m}$ acima do nível do mar, sendo o solo predominante o Latossolo Vermelho Distrófico.

\subsection{Obtenção e preparo de dados}

Foram analisados 23 povoamentoss florestais de 2,5 a 4,5 anos de idade, plantados entre 1997 e 1998, totalizando $12.511,48$ ha. Cada povoamento contou com pelo menos três medições anuais nas parcelas permanentes, que caracterizam o Inventário Florestal Contínuo adotado. A intensidade amostral é de aproximadamente uma parcela retangular de $630 \mathrm{~m}^{2}$ a cada 10 ha.

Os volumes gerados por parcela foram obtidos a partir da equação de volume individual específica para cada povoamento. As alturas dominantes utilizadas foram obtidas pela média aritmética das seis árvores de maior diâmetro, tomado a 1,30 m de altura nas árvores das parcelas. A área basal foi obtida através da soma das áreas transversais de cada árvore, geradas a partir do DAP.

\subsection{Estudo variográfico}

Em cada povoamento, efetuou-se o estudo variográfico, a fim de verificar a estrutura de dependência espacial. Este estudo teve início com a avaliação do semivariograma experimental. Segundo Journel e Huijbregts (1978), a função variograma denominada $2 \gamma(h)$ é a esperança matemática do quadrado da diferença entre pares de pontos separados por uma distância (h):

$$
2 \gamma(h)=E\left\{[Z(x)-Z(x+h)]^{2}\right\}
$$

A metade da função variograma é denominada função semivariograma. O estimador da semivarância é igual à média aritmética das diferenças ao quadrado entre pares de valores observados, em todos os pontos separados pela distância $h$ :

$$
\hat{\gamma}(h)=\frac{1}{2 N(h)} \sum_{i=1}^{N(h)}\left[Z\left(x_{i}\right)-Z\left(x_{i}+h\right)\right]^{2}
$$

em que $\hat{\gamma}(h)$ é a semivariância estimada entre pares de pontos, $N(h)$ é o número de pares de pontos separados por um vetor $h, Z\left(x_{i}\right)$ é o valor da variável regionalizada no ponto $x$ e $Z\left(x_{i}+h\right)$ é o valor no ponto $x_{i}+h$. Essa função permitiu gerar o semivariograma experimental. A partir deste, foi possível ajustar um modelo espacial capaz de reunir toda a informação da estrutura de continuidade espacial. A partir desse ajuste, obtiveram-se os parâmetros do modelo de semivariograma, efeito pepita $\left(\tau^{2}\right)$, contribuição $\left(\sigma^{2}\right)$ e alcance $(\phi)$. Com os parâmetros do modelo espacial de cada característica avaliada, determinou-se o grau de dependência espacial (DE). O grau de dependência espacial é uma relação porcentual entre a variação estruturada $\sigma^{2}$ e o patamar $\left(\tau^{2}+\sigma^{2}\right)$

Existem vários modelos de ajuste aplicáveis a diferentes fenômenos com continuidade espacial. Tais modelos, chamados de "modelos autorizados", devem fornecer soluções estáveis para o estimador linear krigagem. Isso significa que a matriz de co-variância utilizada na krigagem possa ser invertida. Essa condição limita o conjunto de funções usadas na modelagem do semivariograma experimental. Isaaks e Serivastava (1989) descreveram os principais modelos de semivariograma e suas principais características.

R. Árvore, Viçosa-MG, v.31, n.5, p.859-866, 2007 


\subsection{Ajuste dos modelos pelo método dos quadrados mínimos}

O ajuste de modelos espaciais pelo método dos quadrados mínimos ocorreu a partir dos pontos do semivariograma experimental. Esse método consiste em obter os valores dos parâmetros de um modelo que minimizam a soma do quadrado da diferença entre os valores observados e os estimados. A obtenção dos parâmetros do semivariograma pelo método dos quadrados mínimos foi obtida pela minimização da seguinte expressão:

$$
Q(\theta)=\sum_{i=1}^{k}\left[g\left(h_{i}\right)-\gamma\left(h_{i} ; \theta\right)\right]^{2}
$$

em que $\theta$ representa o vetor de parâmetros estimados que definem completamente o semivariograma, sendo cada estimativa denotada por $g\left(h_{i}\right)$; k refere-se ao número de lags do semivariograma experimental; e $\gamma\left(h_{i} ; \theta\right)$ é a semivariância calculada pelo modelo e depende dos parâmetros estimados $\theta$ - após a minimização, a função $g\left(h_{i}\right)$ se torna a estimativa $\hat{\gamma}\left(h_{i}\right)$ de quadrados mínimos. A seleção do melhor modelo para cada povoamento foi efetuada através da comparação entre a soma do quadrado da diferença gerada pelos ajustes dos modelos.

\section{RESULTADOS E DISCUSSÃO}

\subsection{Avaliação da estrutura de continuidade espacial}

Nos Quadros 1, 2 e 3 são apresentados, para as variáveis volume, área basal e altura dominante, os modelos de semivariograma que apresentaram a menor soma do quadrado da diferença para cada um dos povoamentos avaliados e os respectivos parâmetros: efeito pepita $\left(\tau^{2}\right)$, contribuição $\left(\sigma^{2}\right)$ e alcance $(\phi)$. Nesses mesmos quadros se encontram os valores referentes ao grau de dependência espacial (DE\%). Segundo a classificação apresentada por Cambardella et al. (1994) para características de solo, o grau de dependência espacial é forte se DE $>75 \%$, médio quando estiver entre 30 e $75 \%$ e baixo se a DE for menor que $30 \%$.

Quadro 1 - Estimativa dos parâmetros efeito pepita $\left(\tau^{2}\right)$, variação estruturada $\left(\sigma^{2}\right)$, patamar $\left(\tau^{2}+\sigma^{2}\right)$, alcance $(\phi)$ e relação variação estruturada/patamar $(D E \%)$ para volume/ha

Table 1 - Estimate of the nugget effect parameters $\left(\tau^{2}\right)$, structured variation $\left(\sigma^{2}\right)$, sill $\left(\tau^{2}+\sigma^{2}\right)$, range $(\phi)$ and structured variation / sill (DE \%) for volume/ha

\begin{tabular}{|c|c|c|c|c|c|c|c|c|c|c|c|c|c|c|c|}
\hline \multirow[t]{2}{*}{ Povoamento } & \multicolumn{5}{|c|}{$1^{\mathrm{a}}$ medição } & \multicolumn{5}{|c|}{$2^{\text {a }}$ medição } & \multicolumn{5}{|c|}{$3^{\text {a }}$ medição } \\
\hline & Modelo & $\tau^{2}$ & $\sigma^{2}$ & $\phi(m)$ & $D E \%$ & Modelo & $\tau^{2}$ & $\sigma^{2}$ & $\phi(m)$ & $D E \%$ & Modelo & $\tau^{2}$ & $\sigma^{2}$ & $\phi(m)$ & $D E \%$ \\
\hline 1 & Exp & 25,3 & 160,5 & 1985 & 86,4 & Exp & 92,2 & 322,9 & 1985 & 77,8 & Circ & 201,3 & 280,8 & 1708 & 58,3 \\
\hline 2 & Circ & 62,1 & 81,5 & 1867 & 56,8 & Exp & 180,9 & 283,2 & 2191 & 61,0 & Exp & 271,9 & 518,3 & 1637 & 65,6 \\
\hline 3 & Circ & 38,8 & 39,1 & 915 & 50,2 & Esf & 254,2 & 227,5 & 1278 & 47,2 & Circ & 356,4 & 212,3 & 767 & 37,3 \\
\hline 4 & Circ & 24,2 & 235,3 & 2084 & 90,7 & Circ & 223,0 & 514,3 & 2084 & 69,8 & Esf & 391,9 & 651,0 & 2084 & 62,4 \\
\hline 5 & Exp & 0,0 & 28,0 & 992 & 100,0 & Exp & 8,9 & 195,4 & 992 & 95,7 & Esf & 81,8 & 278,0 & 807 & 77,3 \\
\hline 6 & Esf & 0,0 & 27,9 & 352 & 100,0 & Exp & 0,0 & 58,4 & 433 & 100,0 & Circ & 60,8 & 21,2 & 1489 & 25,9 \\
\hline 7 & Esf & 133,1 & 231,3 & 821 & 63,5 & Esf & 194,6 & 104,4 & 1955 & 34,9 & Esf & 406,3 & 121,8 & 1955 & 23,1 \\
\hline 8 & Exp & 13,1 & 53,1 & 2035 & 80,2 & Esf & 51,6 & 133,9 & 2035 & 72,2 & Exp & 80,3 & 281,7 & 2035 & 77,8 \\
\hline 9 & Circ & 145,3 & 180,9 & 1765 & 55,5 & Exp & 196,2 & 293,9 & 3732 & 60,0 & Exp & 387,4 & 289,1 & 4457 & 42,7 \\
\hline 10 & Circ & 43,1 & 13,4 & 2742 & 23,6 & Circ & 126,6 & 0,0 & 2626 & 0,0 & Circ & 414,7 & 0,0 & 2626 & 0,0 \\
\hline 11 & Esf & 100,5 & 127,6 & 828 & 55,9 & Esf & 92,9 & 178,9 & 732 & 65,8 & Exp & 177,2 & 195,6 & 481 & 52,5 \\
\hline 12 & Circ & 66,0 & 115,4 & 2481 & 63,6 & Exp & 88,9 & 481,1 & 2898 & 84,4 & Exp & 231,7 & 542,1 & 2481 & 70,1 \\
\hline 13 & Circ & 200,8 & 59,6 & 2124 & 22,9 & Circ & 291,4 & 183,7 & 2124 & 38,7 & Circ & 386,2 & 567,6 & 2124 & 59,5 \\
\hline 14 & Circ & 131,5 & 166,3 & 1446 & 55,9 & Circ & 422,0 & 131,7 & 1442 & 23,8 & Circ & 669,6 & 36,2 & 696 & 5,1 \\
\hline 15 & Circ & 152,5 & 55,4 & 992 & 26,6 & Esf & 331,7 & 169,3 & 899 & 33,8 & Esf & 339,4 & 120,2 & 2189 & 26,2 \\
\hline 16 & Esf & 90,1 & 27,2 & 1567 & 23,2 & Esf & 240,4 & 276,3 & 591 & 53,5 & Esf & 533,0 & 491,3 & 592 & 48,0 \\
\hline 17 & Circ & 54,3 & 3,4 & 2253 & 5,8 & Esf & 266,0 & 262,5 & 920 & 49,7 & Esf & 412,5 & 780,2 & 970 & 65,4 \\
\hline 18 & Exp & 0,0 & 149,6 & 1439 & 100,0 & Exp & 63,0 & 166,7 & 1100 & 72,6 & Circ & 152,9 & 202,6 & 517 & 57,0 \\
\hline 19 & Circ & 0,0 & 207,2 & 1260 & 100,0 & Circ & 0,0 & 1234,9 & 1260 & 100,0 & Circ & 32,3 & 1881,6 & 1260 & 98,3 \\
\hline 20 & Exp & 17,3 & 164,6 & 1382 & 90,5 & Exp & 224,0 & 609,9 & 1830 & 73,1 & Exp & 401,0 & 728,1 & 1280 & 64,5 \\
\hline 21 & Esf & 79,7 & 187,5 & 1523 & 70,2 & Exp & 0,0 & 713,4 & 1172 & 100,0 & Exp & 9,8 & 1162,8 & 933 & 99,2 \\
\hline 22 & Circ & 138,0 & 17,9 & 1628 & 11,5 & $\operatorname{Exp}$ & 188,3 & 0,0 & 1560 & 0,0 & Esf & 416,2 & 26,1 & 524 & 5,9 \\
\hline 23 & Exp & 328,0 & 0,0 & 2026 & 0,0 & Exp & 644,6 & 0,0 & 2026 & 0,0 & Exp & 919,5 & 284,5 & 347 & 23,6 \\
\hline
\end{tabular}

R. Árvore, Viçosa-MG, v.31, n.5, p.859-866, 2007 
Quadro 2 - Estimativa dos parâmetros efeito pepita $\left(\tau^{2}\right)$, variação estruturada $\left(\sigma^{2}\right)$, patamar $\left(\tau^{2}+\sigma^{2}\right)$, alcance $(\phi)$ e relação variação estruturada/patamar $(D E \%)$ para área basal/ha

Table 2 - Estimate of the nugget effect parameters $\left(\tau^{2}\right)$, structured variation $\left(\sigma^{2}\right)$, sill $\left(\tau^{2}+\sigma^{2}\right)$, range $(\phi)$ and structured variation / sill (DE \%) for basal area/ha

\begin{tabular}{|c|c|c|c|c|c|c|c|c|c|c|c|c|c|c|c|}
\hline \multirow[t]{2}{*}{ Povoamento } & \multicolumn{5}{|c|}{$1^{\mathrm{a}}$ medição } & \multicolumn{5}{|c|}{$2^{\mathrm{a}}$ medição } & \multicolumn{5}{|c|}{$3^{\text {a }}$ medição } \\
\hline & Modelo & $\tau^{2}$ & $\sigma^{2}$ & $\phi(m)$ & $D E \%$ & Modelo & $\tau^{2}$ & $\sigma^{2}$ & $\phi(m)$ & $D E \%$ & Modelo & $\tau^{2}$ & $\sigma^{2}$ & $\phi(m)$ & $D E \%$ \\
\hline 1 & Esf & 0,68 & 2,27 & 1985 & 77,1 & Circ & 1,61 & 2,51 & 1700 & 60,9 & Circ & 1,76 & 2,68 & 1651 & 60,3 \\
\hline 2 & Circ & 1,08 & 1,39 & 1867 & 56,1 & Circ & 2,71 & 2,41 & 1655 & 47,1 & Exp & 2,09 & 4,37 & 1934 & 67,7 \\
\hline 3 & Esf & 0,89 & 1,02 & 1008 & 53,5 & Esf & 2,62 & 2,91 & 1199 & 52,6 & Circ & 3,50 & 2,26 & 768 & 39,3 \\
\hline 4 & Circ & 0,70 & 4,83 & 2084 & 87,4 & Circ & 3,09 & 5,48 & 2084 & 63,9 & Exp & 3,83 & 5,81 & 2084 & 60,2 \\
\hline 5 & Esf & 0,00 & 0,63 & 504 & 100,0 & Exp & 0,00 & 2,71 & 870 & 100,0 & Exp & 0,00 & 3,59 & 779 & 100,0 \\
\hline 6 & Exp & 0,00 & 0,52 & 325 & 100,0 & Circ & 0,51 & 0,14 & 1480 & 21,9 & Circ & 0,66 & 0,11 & 1489 & 14,8 \\
\hline 7 & Esf & 4,01 & 4,29 & 766 & 51,7 & Exp & 2,08 & 1,52 & 1955 & 42,2 & Esf & 2,86 & 1,46 & 1955 & 33,9 \\
\hline 8 & Circ & 0,42 & 1,17 & 1955 & 73,6 & Circ & 0,84 & 1,38 & 1955 & 62,2 & Exp & 0,92 & 2,34 & 1955 & 71,7 \\
\hline 9 & Circ & 2,46 & 2,82 & 2199 & 53,4 & Exp & 1,86 & 4,03 & 3732 & 68,4 & Exp & 2,63 & 1,47 & 3204 & 35,9 \\
\hline 10 & Esf & 1,06 & 0,31 & 2742 & 22,5 & Esf & 1,51 & 0,00 & 2626 & 0,0 & Esf & 2,60 & 0,00 & 2626 & 0,0 \\
\hline 11 & Exp & 1,08 & 3,04 & 834 & 73,8 & Exp & 0,36 & 3,14 & 618 & 89,8 & Exp & 2,09 & 0,97 & 446 & 31,8 \\
\hline 12 & Circ & 1,60 & 2,62 & 2898 & 62,1 & Exp & 1,94 & 3,29 & 2481 & 62,8 & Circ & 3,98 & 3,99 & 2481 & 50,1 \\
\hline 13 & Circ & 2,62 & 1,02 & 2124 & 28,1 & Esf & 0,02 & 3,59 & 644 & 99,4 & Circ & 2,77 & 2,69 & 2124 & 49,3 \\
\hline 14 & Esf & 2,72 & 2,46 & 1446 & 47,5 & Circ & 5,78 & 2,00 & 1446 & 25,7 & Esf & 6,67 & 0,00 & 1389 & 0,0 \\
\hline 15 & Circ & 2,86 & 0,65 & 864 & 18,5 & Circ & 2,69 & 1,69 & 992 & 38,6 & Esf & 1,76 & 1,26 & 858 & 41,8 \\
\hline 16 & Esf & 1,39 & 1,31 & 644 & 48,5 & Esf & 3,64 & 3,06 & 601 & 45,6 & Esf & 4,34 & 4,37 & 597 & 50,1 \\
\hline 17 & Circ & 0,96 & 0,35 & 612 & 26,4 & Exp & 0,76 & 4,58 & 610 & 85,7 & Esf & 3,12 & 5,56 & 875 & 64,0 \\
\hline 18 & Exp & 0,29 & 3,07 & 1439 & 91,4 & Exp & 0,78 & 1,81 & 701 & 69,8 & Circ & 1,59 & 1,83 & 515 & 53,4 \\
\hline 19 & Circ & 0,00 & 4,34 & 1260 & 100,0 & Circ & 0,00 & 12,57 & 1211 & 100,0 & Circ & 0,72 & 13,32 & 1260 & 94,9 \\
\hline 20 & Exp & 0,31 & 3,92 & 1394 & 92,7 & Exp & 2,35 & 4,49 & 1632 & 65,6 & Exp & 3,03 & 5,35 & 1092 & 63,8 \\
\hline 21 & Esf & 1,26 & 2,52 & 1487 & 66,7 & Exp & 0,74 & 5,04 & 1087 & 87,2 & Exp & 0,36 & 7,44 & 918 & 95,3 \\
\hline 22 & Circ & 1,90 & 0,50 & 1628 & 21,0 & Esf & 1,95 & 0,06 & 1446 & 3,0 & Circ & 2,80 & 0,53 & 1395 & 15,9 \\
\hline 23 & Exp & 3,26 & 1,13 & 2114 & 25,7 & Circ & 5,38 & 0,25 & 2114 & 4,4 & Exp & 3,05 & 5,07 & 347 & 62,4 \\
\hline
\end{tabular}

Quadro 3 - Estimativa dos parâmetros efeito pepita $\left(\tau^{2}\right)$, variação estruturada $\left(\sigma^{2}\right)$, patamar $\left(\tau^{2}+\sigma^{2}\right)$, alcance $(\phi)$ e relação variação estruturada/patamar $(D E \%)$ para altura dominante

Table 3 - Estimate of the nugget effect parameters $\left(\tau^{2}\right)$, structured variation $\left(\sigma^{2}\right)$, sill $\left(\tau^{2}+\sigma^{2}\right)$, range $(\phi)$ and structured variation / sill (DE \%) for dominant height

\begin{tabular}{|c|c|c|c|c|c|c|c|c|c|c|c|c|c|c|c|}
\hline \multirow[t]{2}{*}{ Povoamento } & \multicolumn{5}{|c|}{$1^{\mathrm{a}}$ medição } & \multicolumn{5}{|c|}{$2^{\text {a }}$ medição } & \multicolumn{5}{|c|}{$3^{\text {a }}$ medição } \\
\hline & Modelo & $\tau^{2}$ & $\sigma^{2}$ & $\phi(m)$ & $D E \%$ & Modelo & $\tau^{2}$ & $\sigma^{2}$ & $\phi(m)$ & $D E \%$ & Modelo & $\tau^{2}$ & $\sigma^{2}$ & $\phi(m)$ & $D E \%$ \\
\hline 1 & Exp & 0,06 & 1,65 & 1737 & 96,8 & Esf & 0,59 & 0,62 & 1985 & 51,4 & Esf & 0,80 & 0,03 & 1985 & 3,3 \\
\hline 2 & Circ & 0,89 & 0,91 & 1998 & 50,5 & Circ & 1,44 & 1,21 & 2606 & 45,6 & Exp & 0,84 & 0,90 & 1568 & 51,6 \\
\hline 3 & Exp & 1,21 & 0,68 & 1323 & 36,0 & Exp & 1,37 & 2,45 & 1479 & 64,1 & Exp & 0,88 & 1,83 & 692 & 67,5 \\
\hline 4 & Circ & 0,87 & 4,40 & 2084 & 83,5 & Circ & 2,29 & 3,72 & 2084 & 61,8 & Circ & 2,62 & 3,48 & 2084 & 57,0 \\
\hline 5 & Circ & 0,51 & 0,22 & 863 & 30,4 & Circ & 0,45 & 0,96 & 992 & 68,3 & Circ & 0,65 & 1,10 & 820 & 62,7 \\
\hline 6 & Exp & 0,00 & 0,32 & 332 & 100,0 & Exp & 0,10 & 0,11 & 1489 & 52,2 & Exp & 0,15 & 0,50 & 1489 & 76,5 \\
\hline 7 & Circ & 0,98 & 0,63 & 1955 & 39,1 & Circ & 1,12 & 0,29 & 1955 & 20,7 & Exp & 1,55 & 0,69 & 1955 & 30,9 \\
\hline 8 & Exp & 0,48 & 0,63 & 2034 & 57,0 & Circ & 0,84 & 1,30 & 2035 & 60,8 & Circ & 0,61 & 1,91 & 1275 & 75,8 \\
\hline 9 & Circ & 1,81 & 2,67 & 1418 & 59,5 & Exp & 0,94 & 2,56 & 1238 & 73,1 & Esf & 1,59 & 2,28 & 1078 & 58,9 \\
\hline 10 & Circ & 1,18 & 0,07 & 1429 & 5,2 & Exp & 1,01 & 0,32 & 1429 & 23,9 & Circ & 1,61 & 0,29 & 1429 & 15,5 \\
\hline 11 & Circ & 0,19 & 2,08 & 641 & 91,4 & Esf & 0,64 & 2,41 & 598 & 79,0 & Circ & 1,95 & 0,80 & 956 & 29,2 \\
\hline 12 & Exp & 0,57 & 3,60 & 5708 & 86,3 & Exp & 1,01 & 2,62 & 2898 & 72,1 & Circ & 1,65 & 2,83 & 1911 & 63,2 \\
\hline 13 & Esf & 0,50 & 1,11 & 718 & 68,8 & Esf & 1,10 & 3,66 & 533 & 76,8 & Circ & 1,68 & 1,98 & 1230 & 54,0 \\
\hline 14 & Exp & 0,40 & 0,00 & 1446 & 0,0 & Exp & 2,34 & 3,84 & 1446 & 62,1 & Exp & 0,00 & 7,79 & 1446 & 100,0 \\
\hline 15 & Circ & 0,33 & 2,49 & 992 & 88,3 & Circ & 3,15 & 1,76 & 663 & 35,8 & Circ & 3,68 & 2,36 & 2189 & 39,1 \\
\hline 16 & Exp & 1,15 & 0,98 & 2739 & 45,9 & Esf & 1,24 & 0,54 & 2073 & 30,2 & Circ & 1,99 & 0,76 & 2571 & 27,6 \\
\hline 17 & Circ & 0,19 & 0,39 & 764 & 66,9 & Circ & 0,88 & 3,43 & 485 & 79,5 & Esf & 1,24 & 2,77 & 788 & 69,0 \\
\hline 18 & Exp & 0,00 & 2,06 & 1439 & 100,0 & Exp & 0,00 & 2,33 & 1439 & 100,0 & Exp & 0,85 & 1,70 & 1439 & 66,7 \\
\hline 19 & Exp & 0,00 & 3,77 & 1112 & 100,0 & Exp & 0,00 & 4,19 & 1112 & 100,0 & Circ & 0,45 & 4,66 & 985 & 91,1 \\
\hline 20 & Circ & 0,25 & 1,82 & 900 & 87,9 & Esf & 0,98 & 1,76 & 761 & 64,2 & Esf & 1,00 & 2,42 & 424 & 70,7 \\
\hline 21 & Esf & 1,10 & 2,86 & 1577 & 72,3 & Exp & 1,33 & 2,32 & 1586 & 63,6 & Exp & 2,25 & 1,35 & 2625 & 37,6 \\
\hline 22 & Exp & 2,13 & 0,68 & 1628 & 24,0 & Exp & 2,05 & 0,44 & 1628 & 17,8 & Exp & 5,42 & 0,00 & 1560 & 0,0 \\
\hline 23 & Exp & 3,87 & 0,00 & 2026 & 0,0 & Exp & 4,47 & 0,00 & 2026 & 0,0 & Exp & 5,90 & 0,00 & 2026 & 0,0 \\
\hline
\end{tabular}


O efeito pepita aumentou de uma medição para outra nas três características avaliadas. Esse comportamento evidencia que a estrutura de continuidade espacial foi afetada pela idade da floresta, ponto esse relevante para um processo de classificação de sítio com base na estrutura de continuidade espacial. Essa situação mostrou que o efeito competição entre plantas afetou a relação espacial entre as unidades amostrais. As informações dos Quadros 1, 2 e 3 indicam que houve povoamentos sem estrutura de continuidade espacial. A maioria desses povoamentos se concentra basicamente em regiões montanhosas, onde a estrutura do relevo favorece a quebra da continuidade espacial entre as unidades amostrais. Assim, pode-se afirmar que a relação espacial depende de uma gama de fatores que se interagem para proporcionar a estrutura de continuidade espacial.

Analisando as colunas da DE\% das características avaliadas em cada medição, verificaram-se variações de 0 a $100 \%$, ou seja, houve povoamentos sem continuidade espacial e povoamentos onde toda a variação foi explicada pela componente espacial. Na situação em que não houver dependência espacial, deve-se continuar empregando os estimadores da estatística clássica. Esses estimadores foram desenvolvidos com base na independência estatística entre as unidades amostrais (COCHRAN, 1965). Nos povoamentos onde a continuidade espacial foi classificada de média a forte, deve-se considerar a componente espacial nos estimadores de média e variância, proporcionando, assim, precisão sem viés nas estimativas efetuadas (MELLO, 2004).

Os valores de alcance variaram em média de 300 a $3.000 \mathrm{~m}$, aproximadamente, conforme o povoamento e a medição. Esse parâmetro reflete diretamente o grau de continuidade espacial da característica avaliada. Quanto maior for o alcance, mais estruturado espacialmente será a variável regionalizada. $O$ parâmetro alcance relaciona-se de forma inversa com o efeito pepita.
Quanto maior o efeito pepita, menor o alcance, ou seja, há muita variação ao acaso no processo; logo, a semelhança entre as parcelas diminui à medida que aumenta a distância entre elas (SOARES, 2000).

No Quadro 4, encontram-se os porcentuais dos povoamentos que apresentaram forte, média e fraca dependência espacial, em cada uma das três características avaliadas, conforme a classificação de Cambardella et al. (1994). Pelos resultados, observase que a grande maioria dos povoamentos analisados possui de média a forte DE em todas as variáveis estudadas. Observa-se, ainda, que o grau de continuidade espacial dos povoamentoss diminuiu com o aumento da idade do povoamento.

Esse comportamento pode ser explicado pelo efeito do sítio (combinação de variáveis ambientais), onde na primeira medição os povoamentos ainda não são tão afetados pelo sítio e, portanto, são muito semelhantes. À medida que aumenta o processo de competição entre plantas com a idade, o efeito do sítio começa a se manifestar na forma de diferenças que afetam a estrutura da continuidade espacial. Esse processo, entretanto, não foi detectado sistematicamente em todos os povoamentos, havendo situações de aumento e manutenção da dependência espacial.

No Quadro 5 estão representados os aumentos, as diminuições e as manutenções da continuidade espacial entre medições, em cada povoamento e variável analisados. No final foram tabulados os totais de povoamentos que aumentaram, diminuíram e mantiveram a continuidade espacial.

Baseado nesses resultados, observou-se que o número de povoamentos que tendem a diminuir o grau de continuidade espacial foi sempre superior ao número de povoamentos que tendem a aumentar. Outra observação importante foi que o porcentual de povoamentoss cuja continuidade espacial se manteve foi baixo, de 4 a $13 \%$, dependendo da variável e medição analisadas.

Quadro 4 - Percentual de povoamentos com forte, média e fraca dependências espaciais das três medições nas quatro características avaliadas

Table 4-Percentage of stands with strong, average and weak spatial dependence of three measurements for the four appraised characteristics

\begin{tabular}{|c|c|c|c|c|c|c|c|c|c|}
\hline \multirow[t]{2}{*}{ Medição } & \multicolumn{3}{|c|}{ Volume } & \multicolumn{3}{|c|}{ Área basal } & \multicolumn{3}{|c|}{ Altura dominante } \\
\hline & Forte & Méd & Fraca & Forte & Méd & Fraca & Forte & Méd & Fraca \\
\hline 1 & 34,8 & 39,1 & 26,1 & 30,4 & 56,5 & 13,0 & 39,1 & 43,5 & 17,4 \\
\hline 2 & 26,1 & 56,5 & 17,4 & 26,1 & 56,5 & 17,4 & 21,7 & 60,9 & 17,4 \\
\hline 3 & 17,4 & 60,9 & 21,7 & 13,0 & 69,6 & 17,4 & 17,4 & 65,2 & 17,4 \\
\hline
\end{tabular}

R. Árvore, Viçosa-MG, v.31, n.5, p.859-866, 2007 
Quadro 5-Representação do aumento da dependência espacial (+), diminuição (-) e manutenção (=) entre medições (mi) para cada povoamento e variável analisada

Table 5-Representation of the increase in spatial dependence $(+)$, decrease (-) and maintenance (=) among measurements (mi) for each stand and analyzed variable

\begin{tabular}{|c|c|c|c|c|c|c|}
\hline \multirow[t]{2}{*}{ Povoamento } & \multirow{2}{*}{\multicolumn{2}{|c|}{$\begin{array}{c}\text { Volume } \\
\text { m1-m2 m2-m3 }\end{array}$}} & \multicolumn{2}{|c|}{ Area Basal } & \multicolumn{2}{|c|}{ Hdom } \\
\hline & & & $\mathrm{m} 1-\mathrm{m} 2$ & $\mathrm{~m} 2-\mathrm{m} 3$ & $\mathrm{~m} 1-\mathrm{m} 2$ & $\mathrm{~m} 2-\mathrm{m} 3$ \\
\hline C131 & - & - & - & - & - & - \\
\hline C141 & + & + & - & + & - & + \\
\hline $\mathrm{C} 155$ & - & - & - & - & + & + \\
\hline C523 & - & - & - & - & - & - \\
\hline C534 & - & - & $=$ & $=$ & + & - \\
\hline C537 & $=$ & - & - & - & - & + \\
\hline C541 & - & - & - & - & - & + \\
\hline C731 & - & + & - & + & + & + \\
\hline C740 & + & - & + & - & + & - \\
\hline C741 & - & $=$ & - & $=$ & + & - \\
\hline C872 & + & - & + & - & - & - \\
\hline C876 & + & - & + & - & - & - \\
\hline F134 & + & + & + & - & + & - \\
\hline F138 & - & - & - & - & + & + \\
\hline F156 & + & - & + & + & - & + \\
\hline F549 & + & - & - & + & - & - \\
\hline F551 & + & + & + & - & + & - \\
\hline F640 & - & - & - & - & $=$ & - \\
\hline F662 & $=$ & - & $=$ & - & $=$ & - \\
\hline F669 & - & - & - & - & - & + \\
\hline F750 & + & - & + & + & - & - \\
\hline F839 & - & + & - & + & - & - \\
\hline F852 & $=$ & + & - & + & $=$ & $=$ \\
\hline Total + & 9 & 6 & 7 & 7 & 8 & 8 \\
\hline Total - & 11 & 16 & 14 & 14 & 12 & 14 \\
\hline Total & 20 & 22 & 21 & 21 & 20 & 22 \\
\hline$\%+$ & 45 & 27 & 33 & 33 & 40 & 36 \\
\hline$\%-$ & 55 & 73 & 67 & 67 & 60 & 64 \\
\hline
\end{tabular}

Observou-se, ainda, que o volume apresentou grandes oscilações na DE entre as medições analisadas, ou seja, $45 \%$ dos povoamentoss aumentaram a DE entre a primeira e a segunda medição, e entre a segunda e a terceira medição esse número foi de apenas $27 \%$. Com relação à área basal, essa proporção se manteve em 33 e 67\%, em ambos os períodos. Quanto à variável altura dominante, tal proporção também se manteve em cerca de 40 e $60 \%$, em ambos os períodos.

Essa variação da estrutura de continuidade espacial entre medições evidencia que a avaliação da continuidade espacial deve ser efetuada em cada povoamento e em cada uma de suas medições. Caso não haja estrutura de dependência espacial, efetua-se a análise tradicional do inventário florestal. Na presença de continuidade espacial, é recomendado incorporar a componente espacial nas análises de dados (VIEIRA, 2000).

Pelo estudo variográfico efetuado nos 23 povoamentoss florestais, foi possível verificar que, na grande maioria, foi possível fazer uso dos interpoladores espaciais para classificação de sítio com base na característica de interesse. Mediante esses resultados, pode-se pensar em um fluxograma que norteia as análises de inventário e de classificação de sítio para florestas de eucalipto, conforme mostrado na Figura 2.

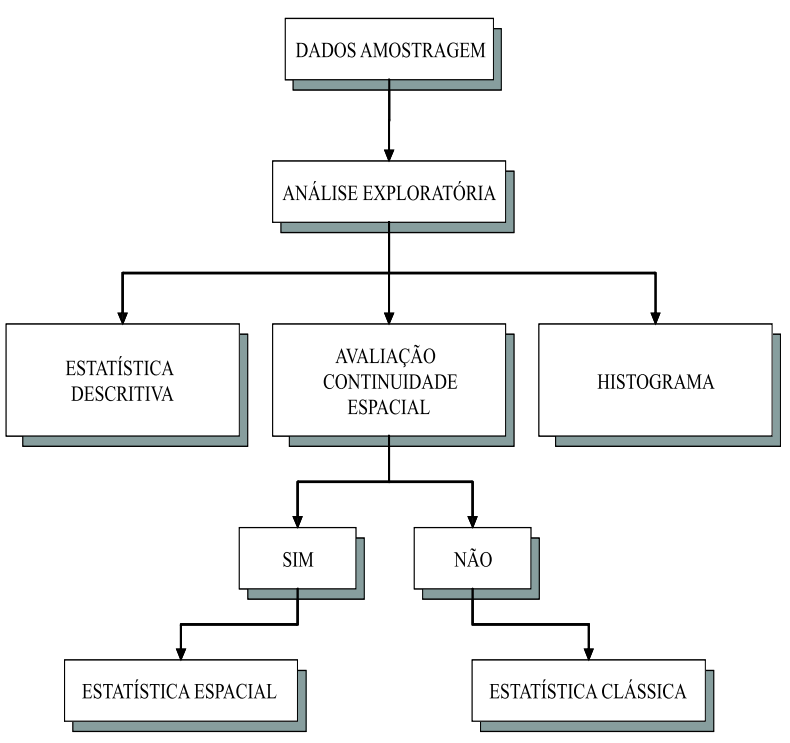

Figura 2 - Fluxograma proposto para uma nova abordagem do inventário florestal e classificação de sítio para florestas de eucalipto.

Figure 2-Flowchart proposed for a new approach of the forest inventory and site index classification for eucalyptus stands.

\section{CONCLUSÕES}

- As características dendrométricas apresentaramse estruturadas espacialmente em alguns povoamentos e em outros não. No caso da existência da estrutura espacializada, sugere-se, no processamento do inventário florestal, considerar a componente espacial. Assim, as parcelas devem ser tratadas de forma dependente e não independente, como preconiza a estatística clássica atual.

- O porcentual de projetos com continuidade espacial foi acima de $70 \%$. A dependência espacial variou de média a forte, entre os projetos avaliados.

R. Árvore, Viçosa-MG, v.31, n.5, p.859-866, 2007 
- Houve diminuição no grau de dependência espacial com a idade do povoamento nas três características avaliadas. Detectaram-se variações na continuidade espacial entre as sucessivas medições, podendo aumentar ou diminuir com o tempo. Porém, a diminuição da continuidade espacial com o tempo foi o padrão que ocorreu com maior frequiência. Pelos resultados deste estudo, a continuidade espacial é um fenômeno que está estritamente ligado com as caracterísitcas do povoamento e com a idade em que este se encontra.

\section{REFERÊNCIAS}

CAMBARDELLA, C. A. et al. Field scale variability of soil properties incentral Iowa soils. Soil Science Society of America jounal, v.58, p.1501-1511,1994.

COCHRAN, W. G. Técnicas de amostragem. Rio de Janeiro, Fundo de Cultura, 1965. 555p.

\section{ENVIRONMENTALSYSTEMS RESEARCH}

INSTITUTE - ESRI. Using ArcGIS

geoestatistical analyst. Redlands: 2001. 300p.

GOLFARI, L.; CASER, R.L.; MOURA, V.P.G

Zoneamento Ecológico Esquemático para Reflorestamento no Brasil.( $2^{\mathrm{a}}$ aproximação). Belo Horizonte: Centro de Pesquisas Florestais da Região do Cerrado, 1978. 66p. (Série Técnica,11).
ISAAKS, E.H.; SRIVASTAVA, R.M. An introduction to applied geostatistics. New York, Oxford University Press, 1989. 560p.

JOURNEL, A.G.; HUIJBREGTS,C.J. Mining geostatistics. London: Academic Press, London, 1978. 600p.

MELLO, J. M. Geoestatística aplicada ao inventário florestal. 2004. 110p. Tese (Doutorado em Recursos Naturais) - Escola Superior de Agricultura "Luiz de Queiroz", Universidade de São Paulo, Piracicaba, 2004.

SCOLFORO, J. R. S.; MELLO, J. M.

Inventário Florestal. Lavras: UFLAFAEPE, 344p. 1997. 344p.

SOARES, A. G. Geoestatística para as ciências da terra e do ambiente. Lisboa: ISTPress, 2000. 186p.

VIEIRA, S. R. Geoestatística em estudos de variabilidade espacial do solo. In: Tópicos em Ciências do Solo. Viçosa, MG: Sociedade Brasileira de Ciência do Solo, 2000.

YAMAMOTO, J. K. Avaliação e classificação de reservas minerais. São Paulo: Universidade de São Paulo, 2001. 226p. 